\title{
Shape Retainment by Utilizing Hysteresis in Piezoelectric Ceramics
}

\author{
By Tadashige $\operatorname{IKEDA}^{1)}$, Tomoki TAKAHASHI ${ }^{2}$, Tomonori UCHIDA ${ }^{2)}$ and Kosei ISHIMURA ${ }^{3)}$ \\ ${ }^{1)}$ Composite Engineering Research Center, Nagoya University, Nagoya, Japan \\ ${ }^{2)}$ Department of Aerospace Engineering, Nagoya University, Nagoya, Japan \\ ${ }^{3)}$ ISAS, JAXA, Sagamihara, Japan
}

(Received June 28th, 2013)

\begin{abstract}
As a future high-precision space structure system, smart structure systems which measure and correct their shape on orbit have been studied. In the smart structure systems piezoelectric ceramics are often used as an actuator. To correct and retain the shape of the structure, electric voltage must be applied continuously. To reduce the amount of electricity usage, a new control method was proposed, where the fact that strain of the piezoelectric ceramics remains without applied voltage after a pulsed voltage input was utilized effectively. In the previous study, the feasibility of the proposed method had been verified for a piezoelectric ceramic plate and a beam with the piezoelectric ceramic plate bonded. The result had shown that the strain of the piezoelectric ceramic plate and the tip displacement of the beam had remained after the pulse, although the beam had vibrated. In this study the strain response for the pulsed voltage was observed for various types of piezoelectric ceramic plates including hard type and soft type, and the vibration of the beam was suppressed by applying a feedback control or a trigonometric shaped pulse input.
\end{abstract}

Key Words: $\quad$ Shape Retainment, Smart Structure, Piezoelectric Ceramics, Hysteresis, Residual Strain

\section{Nomenclature}

$\begin{array}{lll}d_{31} & : \text { piezoelectric constant in the } \\ & \text { longitudinal direction } \\ E & : & \text { electric field } \\ t & : & \text { time in second } \\ T_{\mathrm{C}} & : & \text { Curie point } \\ y & : & \text { ratio of remaining strain to the strain } \\ & \text { during the pulse } \\ Y_{11}{ }^{\mathrm{E}} & : \text { Young's modulus in the longitudinal } \\ & \text { direction } \\ \tan ^{\mathrm{T}} \delta & : & \text { loss factor } \\ \varepsilon_{33}{ }^{\mathrm{T}} / \varepsilon_{0} & : & \text { specific permittivity in the thickness } \\ & & \text { direction }\end{array}$

\section{Introduction}

Recent space telescopes are desired to be larger and to have higher mirror accuracy or pointing accuracy to meet astronomical requirements ${ }^{1-3)}$. However, the performance of such a large space structure is easily affected by errors due to folding-deploying process, thermal cycles, aging, disturbance on orbit, and so on. Accordingly, it is difficult to guarantee their high accuracy during the operation. Moreover, manufacturing error is no longer neglected in such a high precision structure.

To solve these problems, smart structure systems, which measure and correct their configuration, and suppress their vibration on orbit, have been studied ${ }^{4-6}$ ) as a future high-precision space structure system. In the smart structure systems piezoelectric materials are often used as sensors and actuators, because they have relatively high electromechanical conversion efficiency and no sliding part. Recently, Hill et al. ${ }^{7)}$ have developed a large lightweight reflectors comprised of a flexible Kapton film having polyvinylidene fluoride actuators for surface control. They studied optimal grouping of actuators to reduce number of power supplies. Bradford et al. ${ }^{8)}$ have developed an active composite reflector comprised of a graphite face sheet and aluminum honey-comb core composite panel having a network of distributed piezoelectric actuators for vibration and shape control. We have also studied a smart reflector system by using piezoelectric materials to realize high mirror accuracy ${ }^{4)}$.

When shape of a space structure is controlled by the piezoelectric actuators attached to the structural element, electric voltage must be continued to be applied to retain a desired shape. The applied electric power itself is low but the amount of electricity must be accumulated to become big. However, since available energy is limited especially in space, reduction of amount of electricity usage is necessary to save the energy and to extend lifetime of satellites. Moreover, as long as the conventional control method is used, the shape can not be retained, when the control system is down.

Some researchers have studied shape memory ceramics to keep a position of devices such as latching relays and clampers ${ }^{9-11)}$. These devices can be driven by a pulsed voltage and accordingly applied energy can be saved significantly. In these studies, the fact that there are two stable states on zero electric field axis of strain versus electric field curves is applied to alternation of the two positions. These two stable states are caused by hysteresis in phase transition between antiferroelectric phase and ferroelectric phase ${ }^{9)}$ or hysteresis in domain switching between poled domain state and depoled domain state ${ }^{10,11)}$.

Applying the idea of this shape memory ceramics, a new control method was proposed and its feasibility was studied ${ }^{12)}$. 
There it was found that (1) about $100 \mu \varepsilon$ of strain remained after a pulsed voltage, and (2) the residual strain varied depending on the amplitude and period of the pulse. Piezoelectric actuators are often bonded on the structural element. Accordingly, they are stressed during operation. To examine the deformation behavior of the piezoelectric actuators under the stressed condition, a smart beam model was made, where a piezoelectric actuator was bonded on an aluminum beam. From the result of the smart beam, it was also found that (3) even under the stressed condition, bonded on the aluminum alloy beam, the strain remained and accordingly bending displacement of the beam also remained, although the beam vibrated. In this paper, to find an adequate piezoelectric ceramic material, the strain response for a pulsed voltage was examined for some kinds of piezoelectric ceramics including soft type and hard type. Moreover, it was examined if the vibration of the beam after the pulse input could be suppressed by a feedback control or controlling the pulse shape.

The concept of this shape retainment method is reviewed in Section 2. The experimental setup and procedure are shown in Section 3 and the obtained results and discussion are described in Section 4.

\section{Concept of Shape Retainment Method with Zero/Less Electric Field}

Fig. 1 shows a schematic figure of strain (S) versus electric field (E) curve of piezoelectric ceramics for explanation of concept of the present shape retainment method. The origin is defined as the state where the material has been just fabricated and the dipole moments are randomly oriented. By applying a strong DC electric field to it, it polarizes and extends in the direction of the applied electric field. As removing the field, the material is gradually shrinking. However, even after the field is completely removed, residual strain remains (state (a)). If a negative field is applied and the amplitude of the field increases, it continues to shrink until the coercive field $(-E \mathrm{c})$ with no polar character (state (b)). When the negative field increases beyond the coercive field, the material extends again, and it takes a negative polarized state. Then when the negative field is removed and then a positive field increases, it shrinks until the coercive field $(E \mathrm{c})$, and then extends and returns to the positive polarized state, not to the origin. Accordingly the strain versus electric field curve shows a so-called butterfly hysteresis. If the negative electric field is removed from the state (b), the material takes the state (c) different from the state (a) because the orientation of the dipole moments is different from the state (a). Therefore, the strain can take any values (e.g. state (e)) between the state (a) and the state (c) at zero electric field, if the negative electric field is removed from a state (e.g. state (d)) between the state (a) and the state (b). By using this concept, if a shape of a smart structure having a piezoelectric actuator becomes a desired one after removing the electric field, zero electric field is necessary to retain the desired shape. Moreover, even in the case that the shape of the structure deviates slightly from the desired shape due to disturbances or aging, much less electric field is needed to correct the shape than the conventional control method where the electric field must be applied from the state (a)

\section{Experimental Method}

\subsection{Measurement of strain response of piezoelectric ceramic plates}

Schematic diagram of the experimental setup for comparing the strain response of some kinds of piezoelectric ceramic plates against pulsed voltages is shown in Fig. 2. In this study three kinds of ceramic plates (Fuji ceramics, type C-202, C-6, $\mathrm{C}-91 \mathrm{H})$ were used. The specifications of the three specimens are listed in Table 1. The specimens were poled in the thickness direction. Input signals such as rectangular pulses were made by a function generator (NF, WF1974), amplified by an amplifier (Trek, Model 2220), and applied to the piezoelectric ceramic plates. Strain on their surface was measured by a strain gauge. The experiment was carried out in a thermostatic chamber because thermal strain was comparable with the electric strain. A triangular shaped electric field with amplitude of $0.8 \mathrm{kV} / \mathrm{mm}$ was applied to the specimens as a pre-treatment so that the specimens was assumed to be in an equivalent state before the measurements. In this experiment, rectangular pulses with amplitudes of -0.2 $\mathrm{kV} / \mathrm{mm}$ (or -0.4 or $-0.6 \mathrm{kV} / \mathrm{mm}$ ) and $+0.2 \mathrm{kV} / \mathrm{mm}$ (or +0.4 $\mathrm{kV} / \mathrm{mm}$ or $+0.6 \mathrm{kV} / \mathrm{mm}$ ) were applied in series at intervals of $300 \mathrm{~s}$. The period of each pulse was $1 \mathrm{~s}$. For the hard specimen (C-202), the pulses with amplitudes of $\pm 1.2 \mathrm{kV} / \mathrm{mm}$ were also applied since the coercive field is much larger than those of the soft materials. In this case $1.6 \mathrm{kV} / \mathrm{mm}$ was applied for the pretreatment. Next, the amplitude was increased and decreased as $-0.2,-0.4,-0.6,-0.4,-0.2,0.2,0.4,0.6,0.4$, and $0.2 \mathrm{kV} / \mathrm{mm}$, to examine the strain response for continuous variation of the voltage of the pulse input.

\subsection{Measurement of displacement of a smart beam}

Fig. 3 shows the experimental setup for the smart beam. The smart beam model was comprised of an aluminum alloy cantilever beam with a piezoelectric ceramic plate bonded. The size of the cantilever beam was $300 \mathrm{~mm}$ in length, $21 \mathrm{~mm}$ in width, and $1 \mathrm{~mm}$ in thickness. A piezoelectric ceramic plate C-6 with the size of $30 \mathrm{~mm}$ × $20 \mathrm{~mm} \times 1 \mathrm{~mm}$ was bonded so that the end of the piezoelectric ceramic plate was located at $20 \mathrm{~mm}$ from the clamp. Displacement at $20 \mathrm{~mm}$ from the tip was measured with a laser displacement meter (Keyence, LB-02/LB-62) and a strain on the piezoelectric ceramics plate was measured by a strain gauge. In the previous study ${ }^{12)}$ the beam had vibrated after the pulse. To suppress the vibration, a feedback control was applied first. The signal of the laser displacement sensor was filtered and differentiated (MathWorks, SIMULINK with dSPACE, CLP1103), amplified, and applied to the actuator. Then, a trigonometric shaped pulse was applied without feedback control instead of the rectangular shaped pulse.

\section{Results and Discussion}

\subsection{Strain response of piezoelectric ceramics}

Fig. 4 shows the strain response for rectangular pulses of electric field with the amplitude of $\pm 0.2, \pm 0.4, \pm 0.6$, and \pm 1.2 $\mathrm{kV} / \mathrm{mm}$. The time just after the first pulse is define as the 
origin of the time. Negative pulse input generated positive strain because the strain of the surface was induced by $d_{31}$ effect. The strain after a pulse was different from the one before the pulse due to the hysteresis in strain versus electric field curve. The strain remained for $300 \mathrm{~s}$ at least, although the remaining strain became less than a half of the strain during the pulse. The difference between the remaining strains for the positive pulse and the negative pulse for $\mathrm{C}-91 \mathrm{H}$ was the largest, while the remaining strain for C-202 was much smaller than the strain for the others, even though the amplitude of the pulse increased up to $\pm 1.2 \mathrm{kV} / \mathrm{mm}$ as shown in Fig. 4(d). The difference between the remaining strains for the positive pulse and the negative pulse increased with increase in the amplitude of the pulse within the range between the coercive fields. More than $200 \mu \varepsilon$ of remaining strain could be generated by $\mathrm{C}-91 \mathrm{H}$. With respect to the repeatability, mean value of the remaining strain after the pulse varies for \pm 0.2 and $\pm 0.4 \mathrm{kV} / \mathrm{mm}$. This may be because internal state of the specimen changes for the first several cycles. Further investigation is necessary.

Table 2 lists the strain remaining after the 5 th pulse (the $3 \mathrm{rd}$ negative pulse) for C-6 and C-91H. Values in parentheses are ratio of the strain to averaged strain during the pulse. The strain values are calculated by setting the averaged strain for $10 \mathrm{~s}$ just before the 5 th pulse as a baseline. The strain during the pulse was larger than $d_{31} \times E$ because of nonlinearity of the strain versus electric field relationship. The remaining strain seems to decrease as a function of $\log t$ approximately. Fig. 5 shows the ratio of the strain after the 5 th pulse to the strain during the 5 th pulse for $\pm 0.6 \mathrm{kV} / \mathrm{mm}$. It is noted that a logarithmic scale is adopted for the abscissa axis. For the logarithmic time scale, variation of the response seems to increase with time, although it is almost constant over time as shown in Fig. 4 (c) with a normal scale for the abscissa axis. This is because the low frequency variation does not appear explicitly in short time range region in the left of Fig. 5. Approximation curves in the figure are assumed as

$$
y=A-B \log t .
$$

The coefficients of the approximation curve are listed in Table 3. Using this approximation the remaining strain 1 day, 10 days, and 100 days later can be predicted and listed in Table 4, although this may be very rough estimation. The prediction shows that $20 \%$ of the strain during the pulse could remain 100 days later, which is more than $80 \%$ of the strain at $100 \mathrm{~s}$ for C-6 and more than $65 \%$ for C-91H. Even though the energy must be applied to keep the desired strain since the remaining strain decreases, amount of applied energy can be saved compared to the conventional control method.

Fig. 6 shows time history of the strain response of the piezoelectric ceramic plates when the pulses with various amplitudes are applied. The value of the residual strain varied only when the absolute value of the amplitude of the pulses increased in the same sign or the sign of the pulse was altered for all the three types of piezoelectric ceramic plates. Fig. 1 also illustrates the reason. Suppose the piezoelectric actuator is in the state $(e)$. The former means the path $(e) \rightarrow(b) \rightarrow(c)$ or $(\mathrm{e}) \rightarrow(\mathrm{f}) \rightarrow(\mathrm{g})$, respectively, and the latter means the path $(\mathrm{e}) \rightarrow$ $(\mathrm{h}) \rightarrow(\mathrm{e})$. It should be noted that the strain versus electric field curve for the pulse input is different from the quasi-static case because the response of piezoelectric actuator is dependent on variation rate of electric field ${ }^{13)}$.

\subsection{Displacement of a smart beam}

The applied electric field, the tip displacement of the smart beam, and the strain on the piezoelectric ceramic actuator for a pulse of electric field with $-0.6 \mathrm{kV} / \mathrm{mm}$ amplitude and $1 \mathrm{~s}$ period are shown in Fig. 7. The strain and the tip displacement remained although the beam vibrated after the rectangular pulse was applied as shown in Fig. 7(a). To suppress the vibration, a direct velocity feedback control was applied on the rectangular pulse or a trigonometric shaped pulse was applied without feedback control instead of the rectangular pulse. The results are shown in Fig. 7(b) and Fig. 7(c), respectively. It is seen that both the feedback control and the trigonometric shaped pulse were effective for suppressing the vibration. The electric field input for the feedback control vibrated even while the pulse was applied because the feedback control was started just when the pulse input was started to be applied. The trigonometric shaped pulse seems to be better from the viewpoint of response and energy consumption, although the feedback control for the rectangular pulse was not optimized.

\section{Conclusions}

To find an adequate piezoelectric ceramic material for the new shape retainment control method, the strain response for a pulsed voltage was examined for three types of piezoelectric ceramic plates including soft type and hard type. Moreover, it was examined if the vibration of the beam after the pulse could be suppressed by a feedback control or controlling the pulse shape. The results showed that (1) the strain remained after the pulsed voltage and the strain varied when the absolute value of the amplitude of the pulse increased in the same sign or the sign of the pulse was altered for all the three types of piezoelectric ceramic plates, (2) remaining strain of C-91H was the largest with more than $200 \mu \varepsilon$, but for small amplitudes of the pulse input the strain value varied for the first several cycles, (3) the hard material C-202 could not generate enough remaining strain and was inadequate for this control method, and (4) the vibration of the beam with piezoelectric actuator could be suppressed by a feedback control and a trigonometric shaped pulse. These results imply the feasibility of shape control with zero or less applied voltage, although there remain several issues to be solved such as repeatability, reliability, and control method to compensate them.

\section{Acknowledgments}

A part of this study was supported by "Study on Development of High-Precision Large Space Structural System" in the "Science and Engineering Experiments of Strategic Development and Research" of Institute of Space and Astronautical Science, Japan Aerospace Exploration Agency. 


\section{References}

1) Higuchi, K., et al.: Structure of High Precision Large Deployable Reflector for Space VLBI (Very Long Baseline Interferometry) Proceedings of the 50th AIAA/ASME/ASCE/AHS/ASC Structures, Structural Dynamics and Materials Conference, (2009), AIAA-2009-2609, pp. 1-8

2) Agnes, G. S., Dooley, J. and Laboraory, J. P.: Precision Deployable Structures Technology for NASA Large Aperture Missions," Proceedings of Space 2004 Conference and Exhibit, (2004), AIAA-2004-5899, pp. 1-7.

3) Takahashi, T., et al.: The NeXT Mission, Proceedings of SPIE 7011, 2008, 701100 .

4) Okubo, H., et al.: Demonstration Experiment Plan of Ultrahigh-Precision Smart Structure System Using a Scientific Balloon," Proceedings of Scientific Balloon Symposium 2009, (2009), pp. 104-107 (in Japanese).

5) Tanaka, H. and Natrori, M. C.: Shape Control of Space Antennas Consisting of Cable Networks, Acta Astronautica, 55 (2004), pp. 519-527.

6) Ruggiero, E. J. and Inman, D. J.: Gossamer Spacecraft: Recent Trends in Design, Analysis, Experimentation, and Control, Journal of Spacecraft and Rockets, 43 (2006), pp. 10-24.

7) Hill, J. R., Wang, K. W., Fang, W. W. and Quijano, U.: Actuator Grouping Optimization on Flexible Space Reflector, Proceedings of SPIE, 7977 (2011), 797725 pp. 1-12.

8) Bradford, S. C., Agnes, G. S., Bach, V. M. and Wilkie, W. K.: An Active Composite Reflector System for Correcting Thermal Deformations, Proceedings of the 52nd AIAA/ASME/ASCE/AHS/ASC Structures, Structural Dynamics and Materials Conference, (2011), AIAA 2011-1826 pp. 1-8.

9) Uchino, K.: Shape Memory Ceramics, Shape Memory Materials, Otsuka, K. and Wayman, C. M. (ed), Cambridge University Press, Cambridge, (1998), pp. 184-202.

10) Morita, T., Kadota, Y. and Hosaka, H.: Shape Memory Piezoelectric Actuator, Applied Physics Letters, 90 (2007), 082909 pp. 1-3.

11) Kadota, Y., Hosaka, H. and Morita, T.: Field-Induced Strain Memory with Non- $180^{\circ}$ Domain-Reorientation Control, Journal of Korean Physical Society, 57(4) (2010), pp. 902-906.

12) Ikeda, T. and Takahashi, T.: Feasibility Study of Shape Control with Zero Applied Voltage Utilizing Hysteresis in Strain-electric Field Relationship of Piezoelectric Ceramics, Proceedings of SPIE, 8689 (2013), 86890C (8p).

13) Ikeda, T., Yoshida, K. and Ueda, T.: Constitutive Model for Rate Dependent Behavior of Ferroelectric Materials, Proceedings of SPIE, 7978 (2011), 797805 (10p).

Table 1. Specifications of the specimens

\begin{tabular}{|c|r|r|r|}
\hline Specimen & \multicolumn{1}{|c|}{ C-202 } & \multicolumn{1}{c|}{ C-6 } & C-91H \\
\hline Features & $\begin{array}{c}\text { Hard, } \\
\text { High } \mathrm{d} 33\end{array}$ & $\begin{array}{c}\text { Soft, General } \\
\text { Purpose }\end{array}$ & $\begin{array}{c}\text { Soft, } \\
\text { High tan } \delta\end{array}$ \\
\hline$\varepsilon_{33}{ }^{\mathrm{T}} / \varepsilon_{0}[-]$ & 1600 & 2130 & 5800 \\
\hline$d_{31}[\mathrm{pm} / \mathrm{V}]$ & -130 & -210 & -375 \\
\hline$Y_{11}{ }^{\mathrm{E}}[\mathrm{Gpa}]$ & 82 & 62 & 64 \\
\hline $\tan \delta[\%]$ & 0.20 & 1.60 & 3.7 \\
\hline$T_{\mathrm{C}}\left[{ }^{\circ} \mathrm{C}\right]$ & 290 & 295 & 170 \\
\hline Length $[\mathrm{mm}]$ & 40 & 50 & 40 \\
\hline Width $[\mathrm{mm}]$ & 20 & 10 & 20 \\
\hline Thickness $[\mathrm{mm}]$ & 0.5 & 0.5 & 0.5 \\
\hline
\end{tabular}

Table 2. Strain remaining after the pulse $[\mu \varepsilon]$. Values in parentheses are ratio to the averaged strain during the pulse.

\begin{tabular}{|c|c|c|c|c|}
\hline$E[\mathrm{kV} / \mathrm{mm}]$ & \multicolumn{2}{|c|}{-0.4} & \multicolumn{2}{c|}{-0.6} \\
\hline Specimen & C-6 & C-91H & C-6 & C-91H \\
\hline During pulse & 151 & 384 & 257 & 704 \\
& $(1)$ & $(1)$ & $(1)$ & $(1)$ \\
\hline Average & 40.1 & 106 & 62.6 & 244 \\
for 1-2 s & $(0.265)$ & $(0.275)$ & $(0.243)$ & $(0.347)$ \\
\hline Average & 39.2 & 100 & 61.2 & 226 \\
for 10-20 s & $(0.259)$ & $(0.261)$ & $(0.238)$ & $(0.321)$ \\
\hline Average & 38.0 & 96.4 & 59.9 & 211 \\
for 100-200 s & $(0.251)$ & $(0.251)$ & $(0.233)$ & $(0.300)$ \\
\hline
\end{tabular}

Table 3. Coefficients of the approximation curve expressed by Eq. (1).

\begin{tabular}{|c|c|c|c|c|}
\hline $\mathrm{E}[\mathrm{kV} / \mathrm{mm}]$ & \multicolumn{2}{|c|}{-0.4} & \multicolumn{2}{c|}{-0.6} \\
\hline Specimen & C-6 & C-91H & C-6 & C-91H \\
\hline A & 0.270 & 0.273 & 0.245 & 0.345 \\
\hline B & 0.00917 & 0.00996 & 0.00562 & 0.0203 \\
\hline
\end{tabular}

Table 4. Predicted ratio of the strain to the strain during the pulse Values in parentheses are ratio to the strain at $100 \mathrm{~s}$.

\begin{tabular}{|c|c|c|c|c|}
\hline $\mathrm{E}[\mathrm{kV} / \mathrm{mm}]$ & \multicolumn{2}{|c|}{-0.4} & \multicolumn{2}{c|}{-0.6} \\
\hline Specimen & $\mathrm{C}-6$ & $\mathrm{C}-91 \mathrm{H}$ & $\mathrm{C}-6$ & $\mathrm{C}-91 \mathrm{H}$ \\
\hline 100 s later & 0.252 & 0.253 & 0.234 & 0.304 \\
& $(1)$ & $(1)$ & $(1)$ & $(1)$ \\
\hline 1 day later & 0.225 & 0.224 & 0.217 & 0.244 \\
& $(0.893)$ & $(0.884)$ & $(0.929)$ & $(0.803)$ \\
\hline 10 days later & 0.216 & 0.214 & 0.212 & 0.224 \\
& $(0.857)$ & $(0.845)$ & $(0.905)$ & $(0.736)$ \\
\hline 100 days later & 0.207 & 0.204 & 0.206 & 0.203 \\
& $(0.821)$ & $(0.806)$ & $(0.881)$ & $(0.669)$ \\
\hline
\end{tabular}

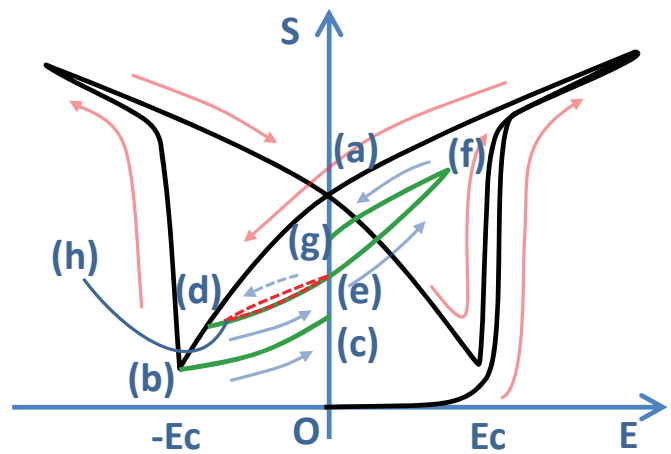

Fig. 1. Explanation of the concept of present control method using a schematic strain $(\mathrm{S})$ versus electric field (E) curve. 


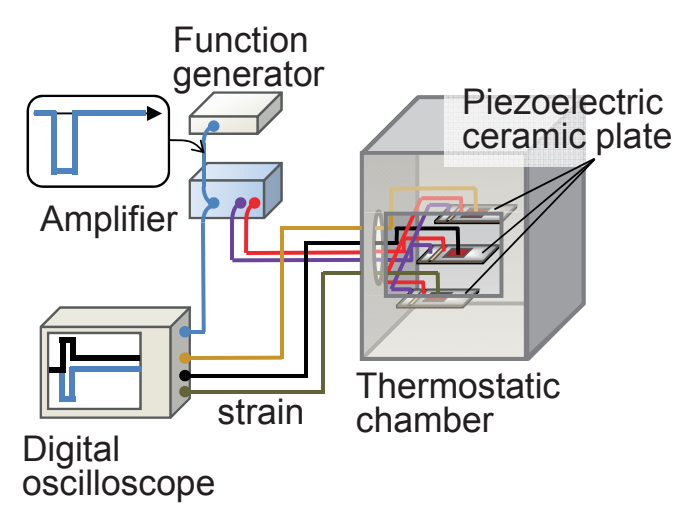

Fig. 2. Schematic diagram of the experimental setup for measurement of strain response of piezoelectric ceramic plates for pulsed voltages.

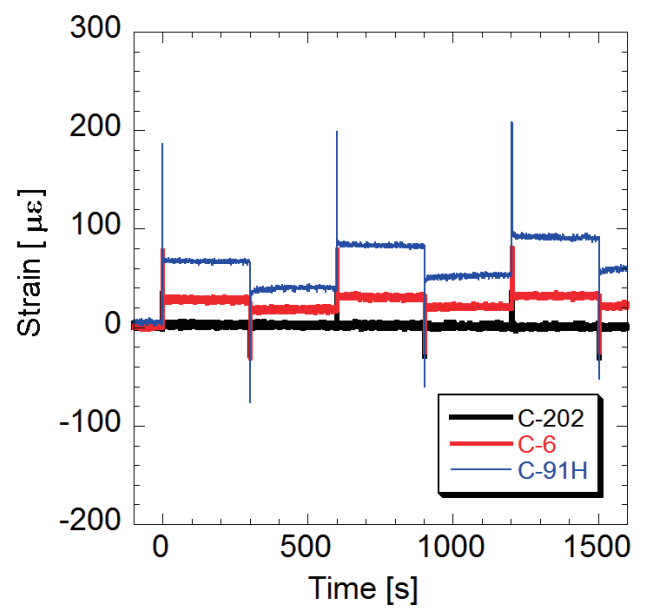

(a) $\pm 0.2 \mathrm{kV} / \mathrm{mm}$

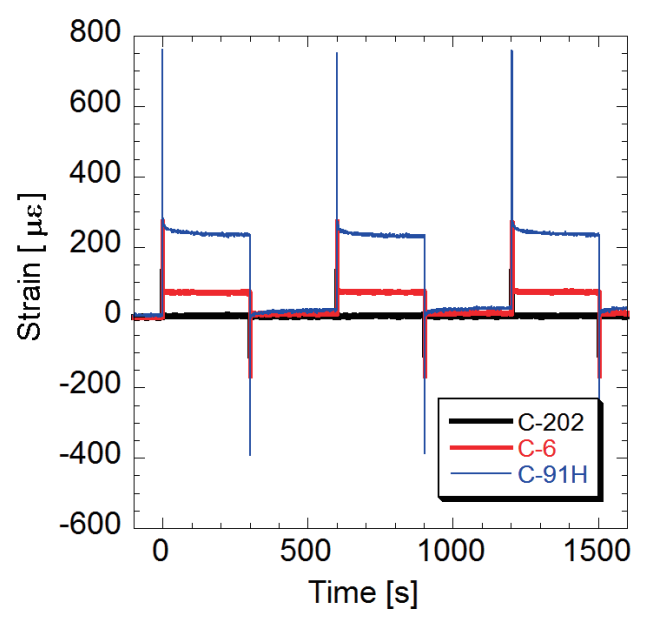

(c) $\pm 0.6 \mathrm{kV} / \mathrm{mm}$

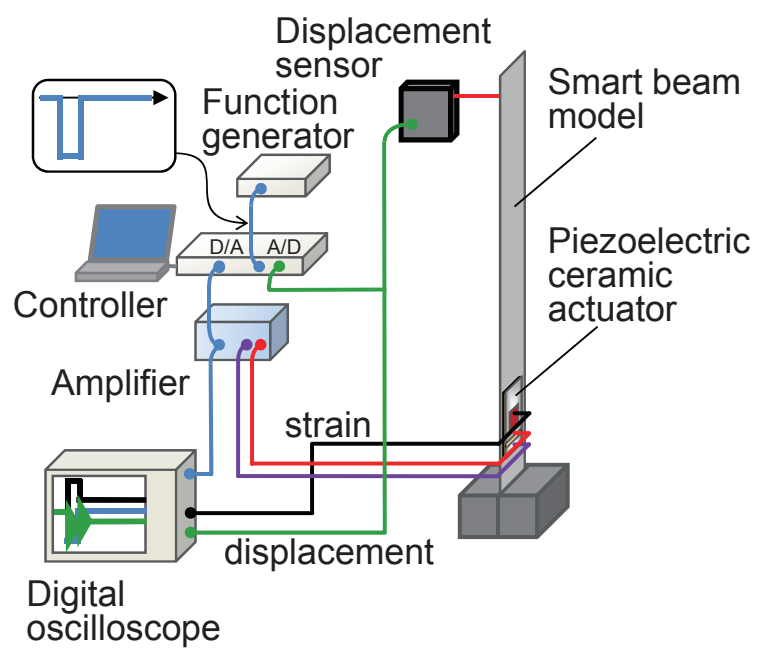

Fig. 3. Schematic diagram of the experimental setup for measurement of response of strain of the piezoelectric actuator and tip displacement of the smart beam model.

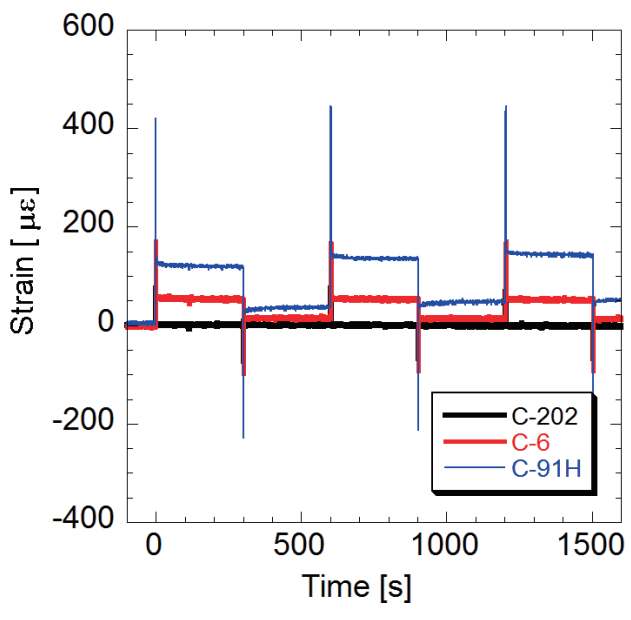

(b) $\pm 0.4 \mathrm{kV} / \mathrm{mm}$

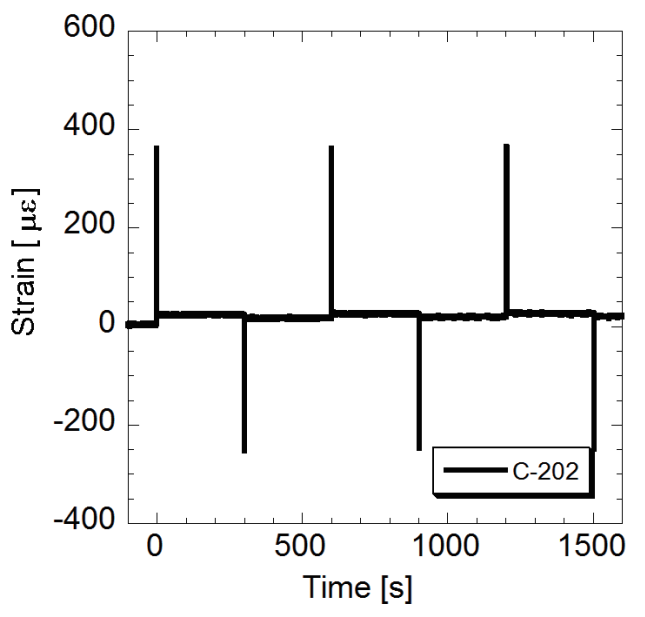

(d) $\pm 1.2 \mathrm{kV} / \mathrm{mm}$

Fig. 4. Strain response of the piezoelectric ceramic plates for pulse inputs. 


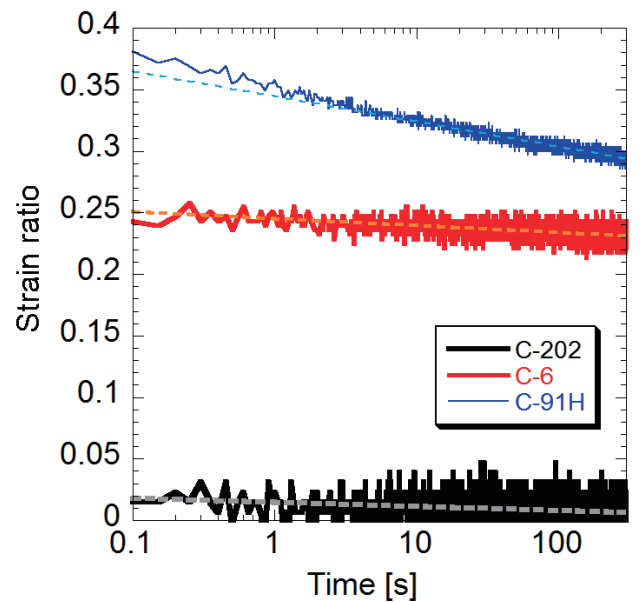

Fig. 5. Ratio of the strain after the 5th pulse to the strain during the 5th pulse for $\pm 0.6 \mathrm{kV} / \mathrm{mm}$.

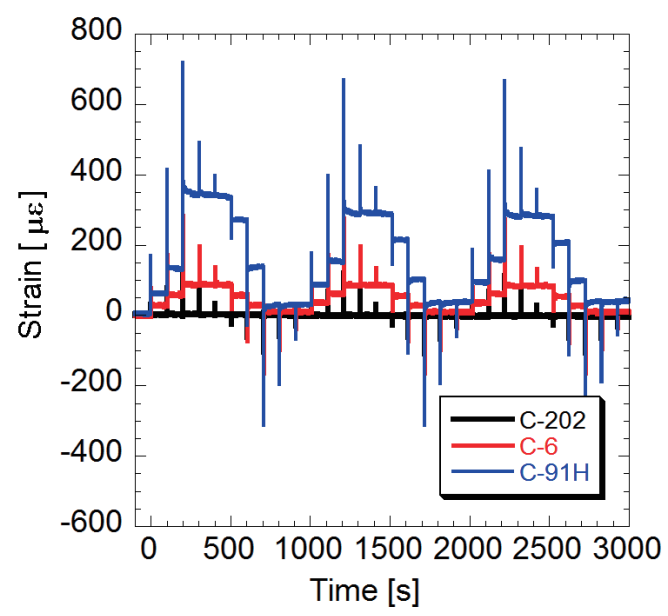

Fig. 6. Strain response of the piezoelectric ceramic plates for pulses whose amplitude increases and decreases.

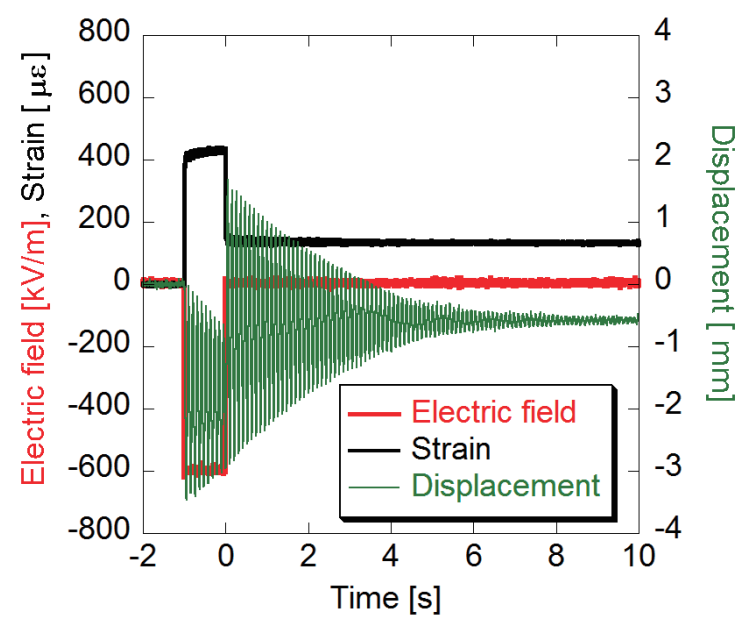

(a) Rectangular pulse

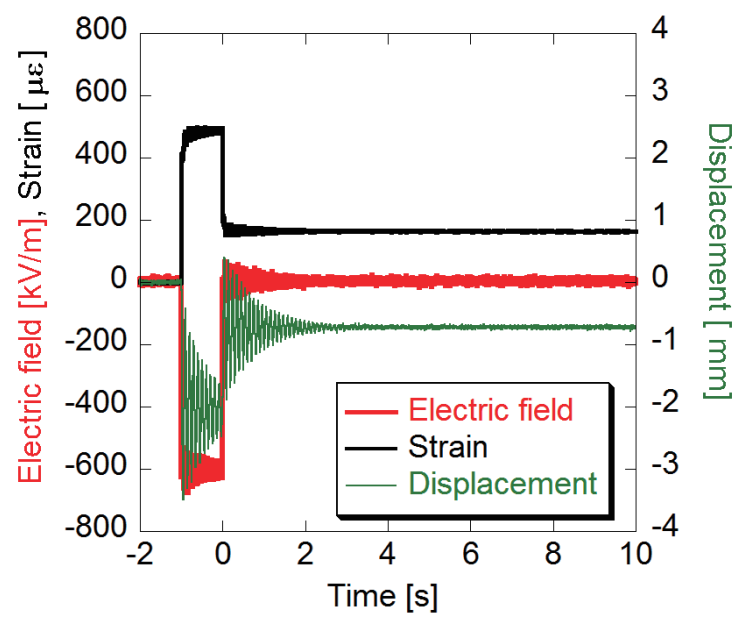

(b) Rectangular pulse with feedback control

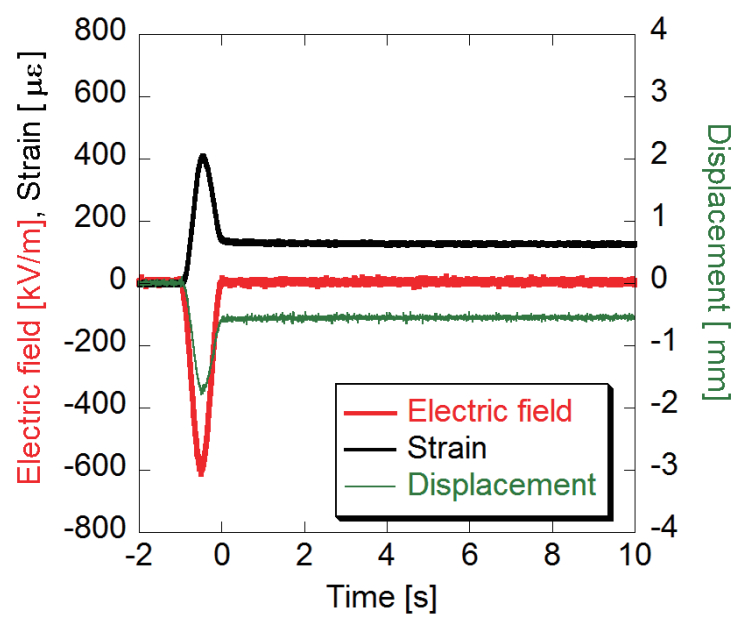

(c) Trigonometric shaped pulse

Fig. 7. Electric field and responses of the strain of the piezoelectric actuator and the tip displacement of the smart beam model. 